\title{
Term-dependent lifetime broadening effect on the 4d photoelectron spectrum of atomic thulium
}

\author{
A G Kochur ${ }^{1}$, I D Petrov ${ }^{1}$, J Schulz ${ }^{2}$ and Ph Wernet $^{3}$ \\ ${ }^{1}$ Rostov State University of Transport Communication, 2 Narodnogo Opolcheniya, \\ Rostov-na-Donu 344038, Russia \\ ${ }^{2}$ Centre for Free-Electron Laser Science at DESY, D-22603 Hamburg, Germany \\ ${ }^{3}$ BESSY, Albert-Einstein-Str. 15, D-12489, Berlin, Germany \\ E-mail: agk@rgups.ru
}

Received 24 July 2008, in final form 16 September 2008

Published 23 October 2008

Online at stacks.iop.org/JPhysB/41/215002

\begin{abstract}
The $4 \mathrm{~d}$ photoelectron spectrum of free thulium atoms is measured and calculated in the single-configuration intermediate-coupling approximation considering multiplet splitting and lifetime broadening of the spectral components due to radiationless transitions following the $4 \mathrm{~d}$ ionization. The individual natural widths of the $4 \mathrm{~d}^{9} 4 \mathrm{f}^{13}$ multiplet components are found to differ considerably, and the experimental profile of the spectrum can be reproduced theoretically only if this effect is taken into account.
\end{abstract}

\section{Introduction}

It is known that the $4 \mathrm{~d}$ photoelectron spectra of the lanthanides with half- and less-than-half-filled $4 \mathrm{f}$ subshell have components with very different natural widths due to the fact that the very rapid $4 \mathrm{~d}-4 \mathrm{f} 4 \mathrm{f}$ super-Coster-Kronig (SCK) transitions are forbidden for the low-ionization-energy highspin components of the $4 \mathrm{~d}^{9} 4 \mathrm{f}^{n}$ multiplet [1-3]. A high-spin state $4 \mathrm{~d}^{9} 4 \mathrm{f}^{n}\left({ }^{2 S_{\max }+1} L\right)$ is the state where the spins of all the $4 \mathrm{f}$ electrons and the spin of the $4 \mathrm{~d}$ vacancy are parallel thus giving the total spin $S_{\max }=n / 2+1 / 2$. The $4 \mathrm{~d}-4 \mathrm{f} 4 \mathrm{f}$ transition would lead to the final state $4 \mathrm{~d}^{10} 4 \mathrm{f}^{n-2} \varepsilon l$ where $\varepsilon l$ is a photoelectron. The maximal possible spin here is $n / 2-1 / 2<S_{\max }$, which means that the transition is forbidden. The natural widths of the high-spin $4 \mathrm{~d}^{9} 4 \mathrm{f}^{n}\left({ }^{2 S_{\max }+1} L\right)$ states are then very small as compared with those of other $4 \mathrm{~d}^{9} 4 \mathrm{f}^{n}$ states for which the $4 \mathrm{~d}-4 \mathrm{f} 4 \mathrm{f}$ SCK transition is the dominant decay pathway. The calculations [1, 3] showed that the difference in individual widths of the $4 \mathrm{~d}^{9} 4 \mathrm{f}^{n}(n \leqslant 7)$ multiplet components is huge, and taking this into account is indispensable in the theoretical description of the $4 \mathrm{~d}$ spectra. The same situation is expected in the $n \mathrm{p}$ photoelectron spectra of the $n \mathrm{~d}$ transition elements $(n=3,4)$ with half- and less-than-half filled $n$ d subshells where the high-spin states $n \mathrm{p}^{5} n \mathrm{~d}^{n}\left({ }^{2 S_{\max }+1} L\right)$ cannot decay by the $n \mathrm{p}-n \mathrm{~d} n \mathrm{~d}$ SCK transition. This has been demonstrated in the cases of the $3 p$ photoelectron spectra of atomic Mn [4] and Mn compounds [5].
As can easily be seen, if the $4 \mathrm{f}$-subshell occupancy in lanthanides or the $n \mathrm{~d}$-subshell occupancy in the $n$ d transition elements is greater than $2 l+1$ then the SCK transitions are not forbidden, and the differences in multiplet component widths are not expected to be dramatic. Nevertheless, it is understood that the individual component widths can be term dependent and considerably different even in those cases. This has been shown, for example, in the calculations of the $L \gamma$ emission spectra of $\mathrm{Tm}$ and $\mathrm{Yb}[6], K \beta$ emission spectra of Fe, Co and $\mathrm{Ni}$ [7], and in $3 \mathrm{p}$ photoelectron spectra of the atomic $\mathrm{Fe}$ and $\mathrm{Co}$ [8], atomic $\mathrm{Ni}$ [9], and $\mathrm{NiCl}_{2}$ [10]. No study on the term dependence of the natural widths in the $4 \mathrm{~d}$ photoelectron spectra of more-than-half-filled $4 \mathrm{f}$ subshell lanthanides has yet been performed.

In this work, we report on the $4 \mathrm{~d}$ photoelectron spectrum of atomic thulium. A theoretical description of the spectrum includes the calculation of the multiplet structure, photoionization cross sections and the broadening of each individual $4 \mathrm{~d}^{-1}$ state due to all possible SCK, Coster-Kronig (CK) and Auger decays.

\section{Experiment}

A beam of free $\mathrm{Tm}$ atoms was produced using a resistively heated oven at approximately $840{ }^{\circ} \mathrm{C}$ [11]. The atoms were ionized by linearly polarized undulator radiation at the $\mathrm{SX}-700$ 
monochromator of the BW3 undulator station at HASYLAB in Hamburg. [12]

The photoelectrons emerging from the interaction region were detected by a SCIENTA SES200 spherical electron analyser. The analyser only accepted electrons with an angle of emission close to the magic angle $54.7^{\circ}$ relative to the polarization axis of the synchrotron radiation. The photoelectron spectra were recorded at a fixed pass energy of $300 \mathrm{eV}$ while scanning the retarding lens voltage of the analyser. For more details of the experimental setup see [13].

The spectrum was taken at a photon energy of $502.5 \mathrm{eV}$ with a total instrumental bandwidth of approximately $0.7 \mathrm{eV}$ (full width at half maximum (FWHM)). The binding energies have been normalized with respect to the Tm 4f lines [14].

\section{Method of calculation}

\subsection{Multiplet structure and photoionization cross sections}

The $4 \mathrm{~d}^{-1}$ multiplets and the $4 \mathrm{~d}$ photoelectron spectra in rare earth atoms were systematically calculated by Demekhin et al using equal widths for all components [15]. They have shown that a good agreement with the experiment can be achieved even in the single-configuration approximation if the configuration interaction effect is accounted for by reducing the electrostatic interaction integrals. The scaling of the interaction integrals accounts for the pressing down of the multiplet components coming from their interaction with higher-lying excited configurations. In this work, we employ the single-configuration intermediate coupling approximation with a scaling factor of 0.67 for $4 \mathrm{~d}-4 \mathrm{f}$ and $4 \mathrm{f}-4 \mathrm{f}$ interaction integrals. The scaling factor was optimized to get the best agreement of the calculated spectrum with the experiment. The radial parts of atomic orbitals and the spin-orbit constants are calculated in the Pauli-Fock approximation [16].

The ground state of the Tm atom is the lower $\mathrm{N}_{5}$ component of the $\mathrm{N}_{45}$ spin doublet, $4 \mathrm{~d}^{10} 4 \mathrm{f}^{132} \mathrm{~F}_{7 / 2}$. The final states of the core after photoabsorption are described in the intermediate-coupling approximation as the expansions over the basic states with the same total momentum $J$ :

$$
|E J\rangle=\sum_{i}\left\langle 4 \mathrm{~d}^{9} 4 \mathrm{f}^{13} \beta_{i} L_{i} S_{i} J|E J>| 4 \mathrm{~d}^{9} 4 \mathrm{f}^{13} \beta_{i} L_{i} S_{i} J\right\rangle .
$$

Here $\beta_{i}$ is an additional quantum number distinguishing between the states with the same $L_{i} S_{i}$ but different genealogy. The energies $E$ and the decomposition coefficients $\left\langle 4 \mathrm{~d}^{9} 4 \mathrm{f}^{13} \beta_{i} L_{i} S_{i} J \mid E J\right\rangle$ were determined by numerical diagonalization of the secular equation matrices. The only empirical parameter entering our calculation of the final-state multiplet structure is the scaling factor for the interaction integrals discussed above.

By employing the methods of $[17,18]$ the cross section of the photoabsorption processes

$$
4 \mathrm{~d}^{10} 4 \mathrm{f}^{132} F_{7 / 2}+h v \rightarrow 4 \mathrm{~d}^{9} 4 \mathrm{f}^{13} E J+\varepsilon l
$$

can be expressed in the form of the product

$$
\begin{aligned}
\sigma^{\varepsilon l}\left({ }^{2} F_{7 / 2} \rightarrow E J\right)=\frac{4 \pi^{2} \alpha a_{0}^{2} h v}{15}(2 J+1) B(E J) \\
\quad \times\left[\left(l\left\|C^{(1)}\right\| \mathrm{d}\right)\langle\varepsilon l|r| 4 \mathrm{~d}\rangle\right]^{2} .
\end{aligned}
$$

Here $l=\{\mathrm{p}, \mathrm{f}\}$ is the photoelectron orbital momentum quantum number, $\alpha$ is the fine structure constant, $a_{0}$ is the Bohr radius, $h v$ is the absorbed photon energy, $\left(l\left\|C^{(1)}\right\| \mathrm{d}\right)$ is the reduced matrix element as defined in [17], and

$$
\langle\varepsilon l|r| 4 \mathrm{~d}\rangle=\int_{0}^{\infty} P_{\varepsilon l}(r) r P_{4 \mathrm{~d}}(r) \mathrm{d} r
$$

is the radial dipole transition integral.

The term $B$ depends on the structure of the final state of the core (1) and is independent of the photoelectron angular momentum.

We found that the radial integrals (4) do not change significantly within the energy range of interest, so they were taken constant. The total cross section for each $4 \mathrm{~d}^{9} 4 \mathrm{f}^{13} E J$ multiplet component was calculated by summing up the partial $4 \mathrm{~d}-\varepsilon \mathrm{p}$ and $4 \mathrm{~d}-\varepsilon$ f cross sections.

\subsection{Component widths}

Consider the decay of the $4 \mathrm{~d}^{9} 4 \mathrm{f}^{13}(E J)$ state into the state $4 \mathrm{~d}^{10} n_{1} l_{1}{ }^{-1} n_{2} l_{2}{ }^{-1}\left(E^{\prime} J^{\prime}\right) \varepsilon l$ through $4 \mathrm{~d}-n_{1} l_{1} n_{2} l_{2}$ radiationless transition, $\varepsilon l$ being Auger, CK or SCK continuous spectrum electron. We consider every possible combination of $n_{1} l_{1}, n_{2} l_{2}$ $=\{4 \mathrm{f}, 5 \mathrm{~s}, 5 \mathrm{p}, 6 \mathrm{~s}\}$. Both $4 \mathrm{~d}^{9} 4 \mathrm{f}^{13}(E J)$ and $4 \mathrm{~d}^{10} n_{1} l_{1}{ }^{-1} n_{2} l_{2}{ }^{-1}\left(E^{\prime} J^{\prime}\right)$ states are expressed as expansions like (1). Then the width of the transition between those two particular terms is

$$
\begin{aligned}
& \Gamma_{4 \mathrm{~d}-n_{1} l_{1} n_{2} l_{2}}\left(E J-E^{\prime} J^{\prime}\right) \\
& =2 \pi \sum_{l}\left|\sum_{i} \sum_{j} C_{i} C_{j} A_{i j}^{\varepsilon l}\left(4 \mathrm{~d}-n_{1} l_{1} n_{2} l_{2}\right)\right|^{2},
\end{aligned}
$$

where $C_{i}=\left\langle 4 \mathrm{~d}^{9} 4 \mathrm{f}^{13} \beta_{i} L_{i} S_{i} J \mid E J\right\rangle$ and $C_{j}=$ $\left\langle 4 \mathrm{~d}^{10} n_{1} l_{1}^{-1} n_{2} l_{2}^{-1} \beta_{j} L_{j} S_{j} J^{\prime} \mid E^{\prime} J^{\prime}\right\rangle$ are the decomposition coefficients, and $A_{i j}^{\varepsilon l}$ is the amplitude of the radiationless transition between the basic $L S J$ states with the ejection of an $\varepsilon l$ electron into the continuum. General expressions for the amplitudes $A_{i j}^{\varepsilon l}$ are given in [19].

Partial width of the $4 \mathrm{~d}^{9} 4 \mathrm{f}^{13}(E J)$ state due to the $4 \mathrm{~d}-n_{1} l_{1} n_{2} l_{2}$ transition is the sum of (5) over the final-state core terms:

$$
\Gamma_{4 \mathrm{~d}-n_{1} l_{1} n_{2} l_{2}}(E J)=\sum_{E^{\prime} J^{\prime}} \Gamma_{4 \mathrm{~d}-n_{1} l_{1} n_{2} l_{2}}\left(E J-E^{\prime} J^{\prime}\right) .
$$

The total $4 \mathrm{~d}^{9} 4 \mathrm{f}^{13}$ (EJ) component width is the sum of (6) over all possible SCK, CK and Auger transitions:

$$
\Gamma(E J)=\sum_{n_{1} l_{1}, n_{2} l_{2}} \Gamma_{4 \mathrm{~d}-n_{1} l_{1} n_{2} l_{2}}(E J)
$$

One may also introduce the partial mean width of the $\operatorname{Tm} N_{5}$ level,

$$
\Gamma_{4 \mathrm{~d}-n_{1} l_{1} n_{2} l_{2}}\left(N_{5}\right)=\sum_{E J} \frac{2 J+1}{g\left(4 \mathrm{~d}^{9} 4 \mathrm{f}^{13}\right)} \Gamma_{4 \mathrm{~d}-n_{1} l_{1} n_{2} l_{2}}(E J),
$$

and the total mean width of the $N_{5}$ level,

$$
\Gamma\left(N_{5}\right)=\sum_{n_{1} l_{1}, n_{2} l_{2}} \Gamma_{4 \mathrm{~d}-n_{1} l_{1} n_{2} l_{2}}\left(N_{5}\right) .
$$

In (8), $g\left(4 \mathrm{~d}^{9} 4 \mathrm{f}^{13}\right)=140$ is the statistical weight of the configuration. 
Table 1. Calculated ionization energies of the $T \mathrm{~m} 4 \mathrm{~d}^{9} 4 \mathrm{f}^{13} E J$ states $(I E)$, cross sections of their production via photoionization of the $\operatorname{Tm} 4 \mathrm{~d}^{10} 4 \mathrm{f}^{132} \mathrm{~F}_{7 / 2}$ state $(\sigma)$, their partial SCK and CK $\left(\Gamma_{4 \mathrm{~d}-n_{1} l_{1} n_{2} l_{2}}\right)$ and total $(\Gamma)$ widths.

\begin{tabular}{|c|c|c|c|c|c|c|c|}
\hline \multirow[b]{2}{*}{$I E, \mathrm{eV}$} & \multirow{2}{*}{$\begin{array}{l}\text { Dominant } \\
\text { basic state }\end{array}$} & \multirow{2}{*}{$\begin{array}{l}\sigma(E J), \\
\text { arbitrary units }\end{array}$} & \multicolumn{4}{|c|}{$\Gamma_{4 \mathrm{~d}-n_{1} l_{1} n_{2} l_{2}}(E J), \mathrm{eV}$} & \multirow[b]{2}{*}{$\Gamma(E J), \mathrm{eV}$} \\
\hline & & & $4 d-4 f 4 f$ & $4 d-4 f 5 s$ & $4 d-4 f 5 p$ & $4 d-4 f 6 s$ & \\
\hline 197.99 & $0.823{ }^{1} \mathrm{P}_{1}$ & 0.017 & 4.782 & 0.323 & 0.773 & 0.032 & 5.939 \\
\hline 197.45 & $0.957{ }^{1} \mathrm{H}_{5}$ & 0.614 & 6.116 & 0.460 & 1.114 & 0.046 & 7.766 \\
\hline 195.79 & $0.689{ }^{3} \mathrm{G}_{3}$ & 0.040 & 5.999 & 0.394 & 0.961 & 0.039 & 7.423 \\
\hline 193.72 & $0.769{ }^{3} \mathrm{P}_{2}$ & 0.380 & 4.943 & 0.314 & 0.755 & 0.031 & 6.074 \\
\hline 192.74 & $-0.626{ }^{3} \mathrm{D}_{3}$ & 0.441 & 5.546 & 0.363 & 0.877 & 0.036 & 6.851 \\
\hline 191.91 & $0.910^{3} \mathrm{G}_{4}$ & 0.582 & 5.976 & 0.381 & 0.933 & 0.037 & 7.357 \\
\hline 191.80 & $0.582{ }^{3} \mathrm{~F}_{2}$ & 0.005 & 4.529 & 0.286 & 0.681 & 0.028 & 5.554 \\
\hline 188.83 & $0.799{ }^{3} \mathrm{D}_{1}$ & 0.166 & 5.070 & 0.343 & 0.817 & 0.033 & 2.549 \\
\hline 188.35 & $0.872{ }^{3} \mathrm{H}_{4}$ & 0.015 & 2.008 & 0.146 & 0.351 & 0.014 & 6.293 \\
\hline 187.10 & $1.000{ }^{3} \mathrm{P}_{0}$ & 0 & 4.757 & 0.230 & 0.704 & 0.028 & 5.809 \\
\hline 187.00 & $0.912{ }^{3} \mathrm{P}_{1}$ & 0.048 & 4.835 & 0.301 & 0.727 & 0.029 & 5.922 \\
\hline 186.76 & $0.674{ }^{1} \mathrm{~F}_{3}$ & 0.477 & 5.558 & 0.373 & 0.900 & 0.037 & 6.898 \\
\hline 186.63 & $0.936{ }^{3} \mathrm{G}_{5}$ & 0.834 & 6.247 & 0.404 & 0.989 & 0.040 & 7.711 \\
\hline 185.90 & $0.617{ }^{3} \mathrm{D}_{2}$ & 0.117 & 4.718 & 0.302 & 0.722 & 0.029 & 5.801 \\
\hline 183.50 & $0.912{ }^{3} \mathrm{~F}_{3}$ & 0.119 & 4.121 & 0.257 & 0.622 & 0.025 & 5.055 \\
\hline 183.25 & $-0.677{ }^{3} \mathrm{~F}_{4}$ & 0.150 & 3.791 & 0.240 & 0.586 & 0.023 & 4.670 \\
\hline 182.75 & $0.946{ }^{3} \mathrm{H}_{5}$ & 0.244 & 2.143 & 0.160 & 0.384 & 0.015 & 2.733 \\
\hline 182.44 & $0.737^{1} \mathrm{D}_{2}$ & 0.268 & 4.286 & 0.262 & 0.622 & 0.025 & 5.226 \\
\hline 180.12 & $0.730{ }^{1} \mathrm{G}_{4}$ & 0.637 & 3.322 & 0.207 & 0.508 & 0.020 & 4.087 \\
\hline 179.22 & $1.000{ }^{3} \mathrm{H}_{6}$ & 1.000 & 1.627 & 0.128 & 0.305 & 0.012 & 2.103 \\
\hline
\end{tabular}

${ }^{\text {a }}$ Principal term in the expansion (1).

The expressions for the amplitudes $A_{i j}^{\varepsilon l}\left(4 \mathrm{~d}-n_{1} l_{1} n_{2} l_{2}\right)$ in (5) contain the electrostatic interaction integrals $R^{k}\left(n_{1} l_{1} n_{2} l_{2}, 4 \mathrm{~d} \varepsilon l\right)$. They were calculated with the core orbitals of the initial configuration $4 \mathrm{~d}^{-1}$. The continuous spectrum wavefunctions $\varepsilon l$ were calculated in the PauliFock approximation in configuration-average potentials of respective final-state two-vacancy configurations built with the core functions of the initial-state configuration $4 \mathrm{~d}^{-1}$. The continuous spectrum electron energies $\varepsilon$ were taken constant within the same $4 \mathrm{~d}-n_{1} l_{1} n_{2} l_{2}$ transition. The integrals $R^{k}\left(n_{1} l_{1} n_{2} l_{2}, 4 \mathrm{~d} \varepsilon l\right)$ were not scaled.

\section{Results and discussion}

Experimental and theoretical Tm 4d photoelectron spectra are presented in figure 1(a). The experimental spectrum is shown with open circles. It is shifted along the vertical axis; its zero intensity level is marked with the horizontal lines. The bar spectrum represents the calculated photoionization cross sections. The profiles of the theoretical spectra were calculated as the sums of the Lorentzians with areas equal to calculated cross sections, and the FWHM equal to calculated components natural widths. The profiles thus obtained were then convoluted with the Gaussians of FWHM $=0.7 \mathrm{eV}$ to account for the experimental broadening. The theoretical profiles were calculated in two ways: (1) with accurate accounting for the individual component natural widths (solid line) and (2) with equal mean natural widths for all the components (dashed line). Calculated natural widths of the spectral components are shown in figure 1(b) and listed in the last column of table 1 .
Table 2. Mean partial widths $\left(\Gamma_{4 \mathrm{~d}-n_{1} l_{1} n_{2} l_{2}}\left(N_{5}\right)\right)$, and total mean width $\left(\Gamma\left(N_{5}\right)\right)$ of the Tm $N_{5}$ level.

\begin{tabular}{ll}
\hline Transition & $\Gamma_{4 \mathrm{~d}-n_{1} l_{1} n_{2} l_{2}}\left(N_{5}\right), \mathrm{eV}$ \\
\hline $4 \mathrm{~d}-4 \mathrm{f} 4 \mathrm{f}$ & 4.3311 \\
$4 \mathrm{~d}-4 \mathrm{f} 5 \mathrm{~s}$ & 0.2887 \\
$4 \mathrm{~d}-4 \mathrm{f} 5 \mathrm{p}$ & 0.6985 \\
$4 \mathrm{~d}-4 \mathrm{f} 6 \mathrm{~s}$ & 0.0281 \\
$4 \mathrm{~d}-5 \mathrm{~s} 5 \mathrm{~s}$ & 0.0037 \\
$4 \mathrm{~d}-5 \mathrm{~s} 5 \mathrm{p}$ & 0.0132 \\
$4 \mathrm{~d}-5 \mathrm{~s} 6 \mathrm{~s}$ & 0.0005 \\
$4 \mathrm{~d}-5 \mathrm{p} 5 \mathrm{p}$ & 0.0124 \\
$4 \mathrm{~d}-5 \mathrm{p} 6 \mathrm{~s}$ & 0.0006 \\
$4 \mathrm{~d}-6 \mathrm{~s} 6 \mathrm{~s}$ & 0.00001 \\
$\Gamma\left(N_{5}\right), \mathrm{eV}$ & 5.3768 \\
\hline
\end{tabular}

Table 1 lists the calculated ionization energies of the Tm $4 \mathrm{~d}^{9} 4 \mathrm{f}^{13} E J$ states, the cross sections of their production via photoionization of the Tm $4 \mathrm{~d}^{10} 4 \mathrm{f}^{132} \mathrm{~F}_{7 / 2}$ state, and their partial SCK and CK widths. The assignment of the $4 \mathrm{~d}^{9} 4 \mathrm{f}^{13}$ multiplet components is given in column 2 of table 1 where the dominant terms in the expansions (1) are listed.

The partial component widths due to the $\mathrm{N}_{5} \mathrm{OO}, \mathrm{N}_{5} \mathrm{OP}$ and $\mathrm{N}_{5} \mathrm{PP}$ Auger transitions are the same for each component, and they coincide with partial $N_{5}$ level widths (8). The partial $N_{5}$ level widths due to all possible radiationless decays and the total mean width of the Tm $N_{5}$ level are listed in table 2 .

It is seen from table 2 that the SCK and CK transitions are responsible for $99.4 \%$ of the total width. Both SCK and CK partial widths are term dependent, and this determines the term dependence of the total widths of the components. One can see from table 1 and figure 1(b) that the individual component 


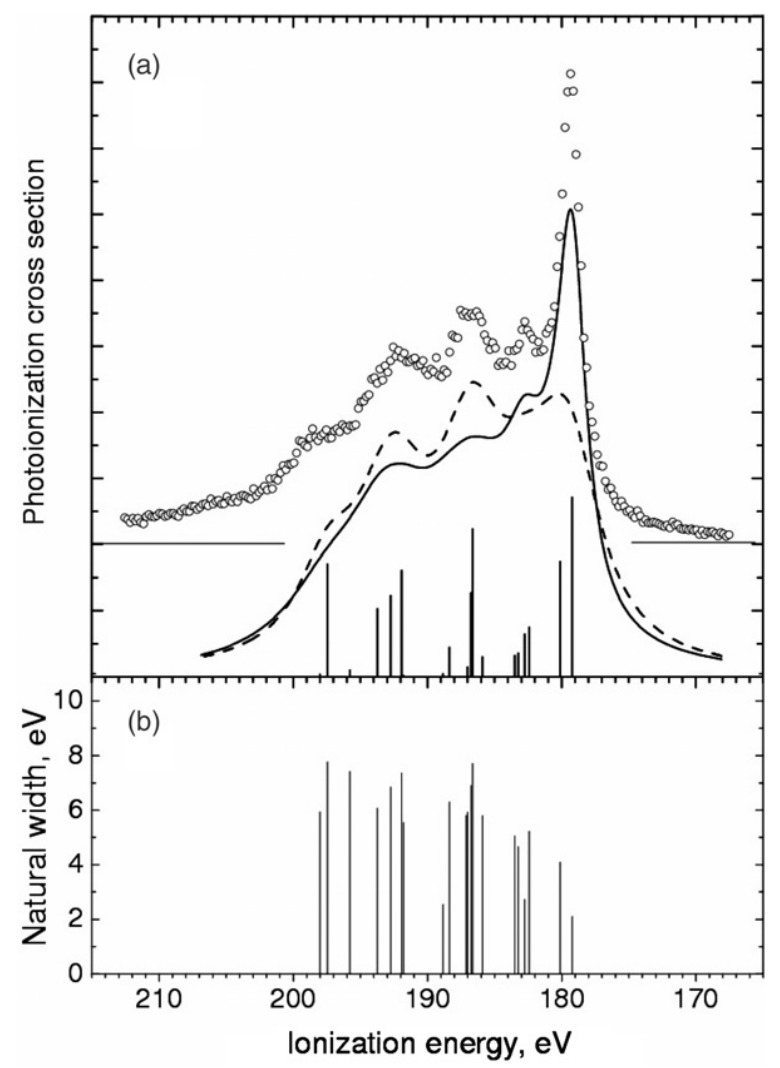

Figure 1. (a) Experimental and calculated 4d photoionization spectrum of atomic thulium. Circles: experiment; bars: calculated photoionization cross sections; solid line: theoretical spectrum with individual components natural widths; dashed line: theoretical spectrum with equal mean components widths. (b) Calculated component natural widths. Assignment of components is given in table 1 .

widths differ significantly - up to a factor of 3.7. Evidently, this is a circumstance that cannot be ignored in the theoretical description of any phenomenon dealing with the $4 d$-hole states in Tm.

The theoretical spectrum calculated with individual component widths (the solid line in figure 1(a)) is in good agreement with the experiment. A broad low-intensity shoulder in the experimental spectrum at about $206 \mathrm{eV}$ which is not reproduced by the calculation comes from the photoionization processes accompanied by satellite shake excitations as discussed in [3].

The spectrum calculated with the same total mean $N_{5}$ level width of $5.38 \mathrm{eV}$ for each component (the dashed line in figure 1(a)) cannot reproduce the profile of the experimental spectrum. We performed the equal-componentwidth calculations taking component widths both greater and less than the calculated one and were never able to get an agreement with the experiment.

\section{Conclusion}

The term dependence of the $4 \mathrm{~d}^{-1}$ states' natural widths is great even in lanthanides with more-than-half-filled $4 \mathrm{f}$ subshell. This must be taken into account in the theoretical description of the $4 \mathrm{~d}$ photoelectron spectra of those elements as well as in any phenomena having to do with the $4 \mathrm{~d}$ vacancies.

\section{References}

[1] Ogasawara H, Kotani A and Thole B T 1994 Phys. Rev. B 50 12332-41

[2] Kochur A G, Sukhorukov V L and Petrov I D 1996 J. Phys. B: At. Mol. Opt. Phys. 39 4565-72

[3] Gerth Ch, Godehusen K, Richter M, Zimmermann P, Schulz J, Wernet Ph, Sonntag B, Kochur A G and Petrov I D 2000 Phys. Rev. A 61022713

[4] von dem Borne A, Johnson R L, Sonntag B, Talkenberg M, Verweyen A, Wernet Ph and Schulz J 2000 Phys. Rev. A 62052703

[5] Tagushi M, Uozumi T and Kotani A 1997 J. Phys. Soc. Japan 66 247-56

[6] Demekhin V F, Sukhorukov V L, Yavna V A, Kulagina S A, Prosandeev S A and Bairachnyi Yu I 1976 Bull. Acad. Sci. USSR Phys. Ser. 40 28-34 translated from 1976 Izvestiva Akademii Nauk SSSR, Seriya Fizicheskaya, USSR 40 255-62

[7] Nemoshkalenko V V, Demekhin V F, Krivitsky V P, Nosachev Yu F, Petrov I D, Yavna V A and Yavna S A 1985 VINITI (USSR) No 3840-85

[8] Tiedtke K, Gerth Ch, Martins M and Zimmermann P 2001 Phys. Rev. A 64022705

[9] Tiedtke K, Gerth Ch, Kanngießer B, Obst B, Zimmermann P, Martins M and Tutay A 1999 Phys. Rev. A 60 3008-12

[10] Okada K, Kotani A, Ogasavara H, Seno Y and Thole D T 2003 Phys. Rev. B 47 6203-6

[11] Ross K and Sonntag B 1995 Rev. Sci. Instrum. 66 4409-33

[12] Björneholm O, Federmann F, Larsson C, Hahn U, Rieck A, Kakar S, Möller T, Beutler A and Fössing F 1995 Rev. Sci. Instrum. 66 1732-4

[13] Wernet Ph, Schulz J, Sonntag B, Godehusen K, Zimmermann P, Grum-Grzhimailo A N, Kabachnik N M and Martins M 2001 Phys. Rev. A 64042707

[14] Wernet Ph, Verweyen A, Schulz J, Sonntag B, Godehusen K, Müller R, Zimmermann P and Martins M 2002 J. Phys. B: At. Mol. Opt. Phys. 35 3887-900

[15] Demekhin V F, Yavna S A, Bairachnyi Yu and Sukhorukov V L 1977 J. Struct. Chem. 18 513-19 translated from 1977 Zhurnal Strukturnoi Khimii (USSR) 18644

[16] Kau R, Petrov I D, Sukhorukov V L and Hotop H 1992 Z. Phys. D 39262

[17] Yutsis A P, Levinson I B and Vanagas V V 1960 Mathematical Apparatus of the Angular Momentum Theory (Vilnius: State Publishing House for Political and Scientific Literature of Lithuanian SSR) p 244

[18] Lindgren I and Morrison J 1982 Atomic Many-Body Theory (Springer Series in Chemical Physics vol 13) (Berlin: Springer) p 469

[19] Kochur A G, Dudenko A I and Petrov I D 2004 J. Phys. B: At. Mol. Opt. Phys. 37 2401-9 